\title{
Short-term oxygen administration restores blunted baroreflex sensitivity in patients with type 1 diabetes
}

\author{
L. Bernardi • M. Rosengård-Bärlund • A. Sandelin • \\ V. P. Mäkinen • C. Forsblom • P.-H. Groop • \\ on behalf of the FinnDiane Study Group
}

Received: 30 July 2010 /Accepted: 22 April 2011 / Published online: 7 June 2011

(C) Springer-Verlag 2011

\begin{abstract}
Aims/hypothesis We hypothesised that the blunted baroreflex sensitivity (BRS) typical of type 1 diabetes is caused by a higher degree of tissue hypoxia in diabetes, and tested whether oxygen increased BRS and ventilation less, equally or more than in healthy control participants (the latter suggesting higher tissue hypoxia). In addition, we also considered the possible interference between oxygen and breathing pattern. Methods In 96 participants with type 1 diabetes and 40 agematched healthy controls, we measured BRS (average of six different standard methods), oxygen saturation, end-tidal carbon dioxide and ventilation changes during spontaneous
\end{abstract}

L. Bernardi and M. Rosengård-Bärlund contributed equally to this study.

L. Bernardi $\cdot$ M. Rosengård-Bärlund $\cdot$ A. Sandelin •

V. P. Mäkinen · C. Forsblom • P.-H. Groop

Folkhälsan Institute of Genetics, Folkhälsan Research Center, University of Helsinki,

Helsinki, Finland

L. Bernardi

Department of Internal Medicine,

IRCCS San Matteo - University of Pavia,

Pavia, Italy

M. Rosengård-Bärlund · V. P. Mäkinen · C. Forsblom •

P.-H. Groop

Department of Medicine, Division of Nephrology,

Helsinki University Central Hospital,

Helsinki, Finland

P.-H. Groop

The Baker IDI Heart and Diabetes Institute,

Melbourne, VIC, Australia

P.-H. Groop $(\bowtie)$

Folkhälsan Research Center, Biomedicum Helsinki (C318b),

University of Helsinki,

PO Box 63, FIN-00014 Helsinki, Finland

e-mail: per-henrik.groop@helsinki.fi and controlled breathing at 15 and six breaths/min, in normoxia and during $5 \mathrm{1} / \mathrm{min}$ oxygen administration.

Results BRS was blunted and blood pressure higher in diabetic participants during spontaneous breathing $(p<0.05)$. BRS increased with oxygen during spontaneous breathing in diabetic $(p<0.001)$ but not in control participants, and with oxygen the difference in BRS was no longer significant. Slow breathing in normoxia restored BRS to a similar extent to giving oxygen. Oxygen increased systolic and diastolic blood pressure, RR interval, heart rate variability, minute ventilation and tidal volume to a greater extent in diabetic patients than in controls, and decreased carbon dioxide similarly to controls. Conclusions/interpretation The increased response to hyperoxia suggests a pre-existing condition of tissue hypoxia that functionally restrains parasympathetic activity in patients with type 1 diabetes. Autonomic abnormalities can be partially and temporarily reversed by functional manoeuvres such as slow breathing or oxygen administration through enhancement of parasympathetic activity and/ or correction of tissue hypoxia.

Keywords Autonomic neuropathy - Baroreflex sensitivity Diabetic retinopathy $\cdot$ Heart rate variability $\cdot$ Hypoxia .

Oxygen - Type 1 diabetes mellitus

$\begin{array}{ll}\text { Abbreviations } \\ \text { BRS } & \text { Baroreflex sensitivity } \\ \text { COPD } & \text { Chronic obstructive pulmonary disease } \\ \text { SBP } & \text { Systolic blood pressure } \\ \text { SDNN } & \text { Standard deviation of all RR intervals }\end{array}$

\section{Introduction}

Although the impairment of neural regulation in diabetes has been well documented and defined during the past four 
decades, the precise conditions in which such alterations occur and develop are still unclear. In particular, the extent and relative proportion to which the abnormalities observed are the result of morphological neuropathy or are functional in nature remain unclear.

Defining the threshold between functional and organic alteration could be of great practical importance. While many clinical trials failed to demonstrate improvements in diabetic autonomic neuropathy based on various rationales that assumed neural damage $[1,2]$, interventions based on the idea of functional abnormalities (e.g. physical training) proved to be effective $[3,4]$. On a shorter timescale, it was shown that even a simple procedure such as slow breathing, an intervention known to reduce sympathetic drive and increase baroreflex sensitivity (BRS) [5, 6], increased BRS in diabetic patients with type 1 diabetes and subnormal baseline values [7]

Several possibilities may explain why cardiovascular modulation can be functionally impaired in patients with type 1 diabetes. These involve insulin treatment, hyperglycaemia, abnormal glycaemic control, previous hypoglycaemia and s-cortisol [8-12]. Additionally, hypoxia has recently attracted the attention of diabetologists as it seems to be implicated in the origin of important complications, such as diabetic nephropathy and diabetic retinopathy [1315]. During acute exposure to high altitude, hypoxia induces direct vasodilation and reflex sympathetic activation, chemoreflex activation and a reduction in BRS [16].

In this study, we tested the hypothesis that BRS, an established prognostic index in cardiovascular diseases [17-19], is restrained by hypoxia in patients with type 1 diabetes. We reasoned that if this were the case, short-term inhalation of oxygen should restore BRS, and diabetic participants would be more responsive to the same intervention than healthy control participants. Conversely, in the presence of a neural lesion, and also if hypoxia were not responsible for the reduced BRS, diabetic individuals would not be responsive to hypoxia or else would respond to an extent similar to but not greater than the healthy participants. Several studies have shown that oxygen administration reduces heart rate and increases indices of heart rate variability related to parasympathetic activity [20-23]. Finally, oxygen administration increases ventilation by a dose-dependent stimulation of the parasympathetic lung afferents [24, 25]. Two studies reported an increase in BRS in healthy participants [21] or patients with chronic obstructive pulmonary disease (COPD) [22], but none measured ventilatory changes or BRS responses to hyperoxia in diabetes. Inhalation of oxygen may be a simple intervention to test the hypothesis of a resting hypoxic condition in diabetes, and test the efficiency of the autonomic function with a simple clinical test.

\section{Methods}

Participants We studied 96 participants with type 1 diabetes and 40 age-matched healthy controls. The participants were recruited through the register of The Social Insurance Institution that comprises all patients entitled to special reimbursement of insulin or antidiabetic medication in Finland. Selection criteria were diabetes (E10 in ICD-10; www.who.int/classifications/icd/en/) diagnosed before the age of 35 years, and age $18-35$ years at the time of inclusion.

Type 1 diabetes was defined as C-peptide deficiency $(<0.03 \mathrm{nmol} / \mathrm{l})$ and initiation of permanent insulin treatment within 1 year after the diagnosis of diabetes. None of the patients showed clinical signs of cardiovascular disease. However, six patients were given laser treatment because of diabetic retinopathy.

Nineteen patients received antihypertensive medication (14 with an ACE inhibitor, one with a combination of an ACE inhibitor, a calcium channel blocker and a diuretic, one with a combination of an ACE inhibitor and a calcium channel blocker, and three with angiotensin-2 receptor blockers). The healthy control participants were recruited by e-mail advertisements among university students and staff. Only individuals with a normal fasting glucose level and without first-degree relatives with diabetes mellitus were included. Before participation, all participants gave their written informed consent. The study protocol was approved by the Ethics Committee of Helsinki University Hospital, and the study was carried out in accordance with the principles of the Declaration of Helsinki as revised in 2000.

The participants underwent a clinical examination, resting ECG, laboratory testing, overnight urine collections, and standard autonomic function evaluation by four cardiovascular tests: the expiration/inspiration ratio of $\mathrm{RR}$ interval during slow deep breathing, the maximum/minimum 30:15 ratio of $R R$ interval during a Valsalva manoeuvre and active standing, and the systolic blood pressure (SBP) response to standing. Cardiovascular autonomic neuropathy was defined as the presence of two or more abnormal tests [26].

Each participant completed a detailed questionnaire on lifestyle, smoking habits and family history.

Protocol All participants were investigated in a quiet room, at a temperature between $19^{\circ} \mathrm{C}$ and $23^{\circ} \mathrm{C}$, between 08:00 and 14:00 hours. The participants received instructions to refrain from alcohol for $36 \mathrm{~h}$, and caffeinated beverages and cigarettes for $12 \mathrm{~h}$ prior to the examination. A light meal was permitted $2 \mathrm{~h}$ before testing. If a participant reported or measured symptoms or values of hypoglycaemia in the previous $24 \mathrm{~h}$, the test was postponed. The ECG was 
recorded using a bipolar precordial lead. Continuous blood pressure was monitored with a Finapres 2300 digital plethysmograph (Ohmeda, Louisville, CO, USA) from the middle finger of the right arm held at heart level. Two respiratory signals were obtained by inductive plethysmography (Z-rip; Pro-Tech, Mukilteo, WA, USA), from belts positioned around the chest and the abdomen. Pulse oximetry and expired carbon dioxide partial pressure (Cosmo; Novametrix, Wallingford, CT, USA) were also obtained.

The signals were simultaneously recorded in the supine position during $5 \mathrm{~min}$ spontaneous breathing, during $2 \mathrm{~min}$ controlled breathing at a frequency similar to normal spontaneous breathing ( 15 breaths/min), and during $2 \mathrm{~min}$ slow deep breathing at the rate of six cycles/min. Subsequently, the participants repeated the entire protocol while breathing $5 \mathrm{l} / \mathrm{min}$ oxygen. Signal recordings started after the first $5 \mathrm{~min}$ of oxygen administration to allow stabilisation of oxygen saturation and ventilation. The sequence of breathing rate (spontaneous, $15 / \mathrm{min}$ and 6/min) was randomised within each session (normoxia and hyperoxia). All signals were simultaneously acquired on a personal computer with an analogue-to-digital converter with a 12-bit resolution at a sampling rate of $200 \mathrm{~Hz}$ (WinAcq data acquisition system; Absolute Aliens Ltd, Turku, Finland).

Assessment of BRS From the original data, the time series of RR interval (from each of two consecutive $\mathrm{R}$ waves of the ECG) and SBP were obtained. Previous studies have shown a poor correlation between different indices of BRS, while, on the other hand, no method has shown clear superior performance over the other [27]. Accordingly, we computed a set of six different tests and used their average $[7,28]$.

BRS was determined from spontaneous fluctuations in the RR interval and SBP during the spontaneous, $15 / \mathrm{min}$ and $6 / \mathrm{min}$ recordings using the positive and negative sequence methods [29], the alpha coefficient in the lowand high-frequency bands and its average [30], and the transfer function technique [31]. In the sequence methods, BRS was estimated by identifying spontaneously occurring sequences of three or more consecutive heartbeats in which both the SBP and the subsequent RR intervals changed in the same direction. The minimum criteria for change were $1 \mathrm{mmHg}$ for SBP and $5 \mathrm{~ms}$ for the RR intervals. For identified positive and negative sequences with a correlation coefficient between the RR intervals and the SBP exceeding 0.85 , the regression slopes (the slope of the regression line between SBP and RR intervals) were calculated, and the average was taken as a measure of BRS positive and negative slopes, respectively. The other four BRS methods were calculated by autoregressive uni- and bivariate spectral analysis [5]. The alpha coefficient was calculated as the square root of the ratio of the powers of RR intervals and SBP in the low frequency range $(0.04-0.15 \mathrm{~Hz})$ and in the respiratory $(0.15-0.40 \mathrm{~Hz})$ high-frequency range when coherence was greater than 0.5 and the phase difference between the SBP and RR intervals was negative. In the transfer function method, BRS was calculated as the average value of SBP-RR cross-spectrum divided by the SBP spectrum in the lowfrequency range $(0.04-0.15 \mathrm{~Hz})$, when coherence exceeded 0.5 .

Analysis of respiration The signals from the inductive plethysmographic belt signals were analysed by an automated program with interactive supervision, to identify for each breath the positive and negative respiratory peaks, together with the respiratory period. The sum of the signals obtained by the two belts was taken as a relative index of tidal volume. The same program automatically identified the end-expiratory (end-tidal) value in the carbon dioxide signal. Using the inductive belt data, a semi-quantitative intra-subject analysis of ventilation could be obtained, by comparing the relative changes in tidal volume and minute ventilation induced by oxygen inhalation or different breathing patterns. Although the device used for the present study does not allow us to obtain tidal volume and minute ventilation in absolute values ( $\mathrm{ml}$ and $1 / \mathrm{min}$, respectively), we took advantage of the strong linear relationship between tidal volume and the inductive belt signals [32], enabling us to obtain ventilation in relative units. The limitation of the semi-quantitative analysis is compensated by the lack of interference with the spontaneous breathing, typical of mouthpieces [33]. We therefore set the minute ventilation obtained during spontaneous breathing in normoxia (our baseline) as $100 \%$ in each participant, and calculated the minute ventilation or tidal volume in percentage changes from that value for each recording.

Laboratory tests Venous blood samples were obtained after a light breakfast and were analysed for $\mathrm{HbA}_{1 \mathrm{c}}$, lipids and serum creatinine. $\mathrm{HbA}_{1 \mathrm{c}}$ concentrations were determined by an immunoturbidimetric immunoassay (Medix Biochemica, Kauniainen, Finland). Serum lipids (cholesterol, triacylglycerols and HDL-cholesterol) and creatinine were measured by enzymatic methods. Urinary AER was measured from three consecutive timed urine collections, one $24 \mathrm{~h}$ and two overnight collections. Normal AER was defined as values persistently $<20 \mu \mathrm{g} / \mathrm{min}$ or $<30 \mathrm{mg} / 24 \mathrm{~h}$, microalbuminuria as AER $\geq 20<200 \mu \mathrm{g} / \mathrm{min}$ or $\geq 30<300 \mathrm{mg} / 24 \mathrm{~h}$, and macroalbuminuria as AER $\geq 200 \mu \mathrm{g} / \mathrm{min}$ or $\geq 300 \mathrm{mg} / 24 \mathrm{~h}$ in at least two out of three urine collections [34]. 
Statistical analyses Data are presented as mean $\pm 95 \%$ CI, unless differently stated. A global index of heart rate variability was assessed using the standard deviation of the RR interval (SDNN), as this variable has a more normal distribution as compared with other indices of variability (e.g. variance). Differences between the two groups and between conditions were expressed as mean \pm 95\% CI. Statistical differences between participants with type 1 diabetes and healthy control participants and interventions $(6 / \mathrm{min}$ vs $15 / \mathrm{min}$ controlled breathing and oxygen) were tested using a linear normal model. As outcome, we modelled the different continuous variables (BRS and blood pressures), and included conditions (normoxia/hyperoxia), breathing patterns (spontaneous, controlled at 15 breaths $/ \mathrm{min}$ and 6 breaths $/ \mathrm{min}$ ) and participant group (healthy control/diabetic) as categorical covariates. For each continuous variable, we assessed the interactions between conditions, groups and breathing patterns [35]. The Sheffé test was used to test for significances between different breathing rates. All tests were repeated after adjusting for the effect of age. Linear regression analysis was used to test for association between variables. Statistical significance was defined as a $p$ value $\leq 0.05$.

\section{Results}

The clinical characteristics of the participants studied are shown in Table 1. The diabetic participants studied had reasonable glycaemic control, one participant had macroalbuminuria, six had microalbuminuria, 19 had one abnormal autonomic test (orthostatic hypotension was abnormal in two participants), and deep breathing was also reduced in five controls. Average values of mean RR interval, systolic and diastolic blood pressure, and oxygen saturation are shown in Table 2 with the oxygen-induced differences and their estimates. The effects of oxygen on BRS are shown in Fig. 1.

Resting data and effect of respiratory manoeuvres As expected, the patients with diabetes had lower $(p<0.05)$ $\mathrm{BRS}$, and a trend to a reduced RR interval and heart rate variability, compared with the control participants. In addition, diabetic participants had higher resting systolic and diastolic blood pressures $(p<0.05)$. All other variables, including resting oxygen saturation, were similar to controls (Table 2).

During slow breathing, BRS increased more in diabetic than in control participants, and as a result the statistical
Table 1 Clinical characteristics and laboratory measurements of participants with type 1 diabetes and healthy control participants (mean \pm SEM)
DBP, diastolic blood pressure

${ }^{\text {a }}$ Data obtained from 24 control participants

\begin{tabular}{|c|c|c|c|}
\hline Characteristic & Type 1 diabetes & Controls & $p$ value \\
\hline$n$ & 96 & 40 & \\
\hline Sex (men/women) & $54 / 42$ & $19 / 21$ & NS \\
\hline Age (years) & $31.5 \pm 0.6$ & $31.0 \pm 1.1$ & NS \\
\hline Duration of diabetes (years) & $13.5 \pm 0.4$ & 0 & \\
\hline Age at onset (years) & $17.9 \pm 0.6$ & - & \\
\hline BMI $\left(\mathrm{kg} / \mathrm{m}^{2}\right)$ & $25.7 \pm 0.4$ & $23.8 \pm 0.6$ & $<0.05$ \\
\hline WHR & $0.87 \pm 0.1$ & $0.86 \pm 0.01$ & NS \\
\hline Current smokers, $n(\%)$ & $19(19.7)$ & $5(12.6)$ & \\
\hline Antihypertensive treatment, $n(\%)$ & $19(19.8)$ & 0 & \\
\hline Insulin dose (U/day) & $60.2 \pm 2.6$ & 0 & \\
\hline Laser-treated retinopathy, $n(\%)$ & $6(6.2)$ & 0 & \\
\hline Microalbuminuria, $n(\%)$ & $6(6.2)$ & 0 & \\
\hline Macroalbuminuria, $n(\%)$ & $1(1)$ & 0 & \\
\hline $\mathrm{HbA}_{1 \mathrm{c}}$ & & & $<0.001$ \\
\hline$\%$ & $8.05 \pm 0.12$ & $5.25 \pm 0.04^{\mathrm{a}}$ & \\
\hline $\mathrm{mmol} / \mathrm{mol}$ & $64.5 \pm 1.3$ & $33.8 \pm 0.4^{\mathrm{a}}$ & \\
\hline Total cholesterol $(\mathrm{mmol} / \mathrm{l})$ & $4.55 \pm 0.08$ & $4.35 \pm 0.15^{\mathrm{a}}$ & NS \\
\hline HDL-cholesterol (mmol/l) & $1.63 \pm 0.05$ & $1.62 \pm 0.09^{\mathrm{a}}$ & NS \\
\hline Triacylglycerol (mmol/l) & $1.21 \pm 0.08$ & $0.85 \pm 0.07^{\mathrm{a}}$ & $<0.05$ \\
\hline Urinary AER (mg/24 h) & $23.04 \pm 11.05$ & $6.72 \pm 1.08^{\mathrm{a}}$ & NS \\
\hline Serum creatinine $(\mu \mathrm{mol} / \mathrm{l})$ & $68.6 \pm 1.2$ & $72.2 \pm 2.7^{\mathrm{a}}$ & NS \\
\hline Clinic SBP (mmHg) & $129.7 \pm 1.2$ & $120.9 \pm 2.7$ & $<0.005$ \\
\hline Clinic DBP (mmHg) & $77.1 \pm 0.9$ & $75.1 \pm 1.3$ & NS \\
\hline Autonomic score & $0.20 \pm 0.04$ & $0.12 \pm 0.05$ & NS \\
\hline
\end{tabular}




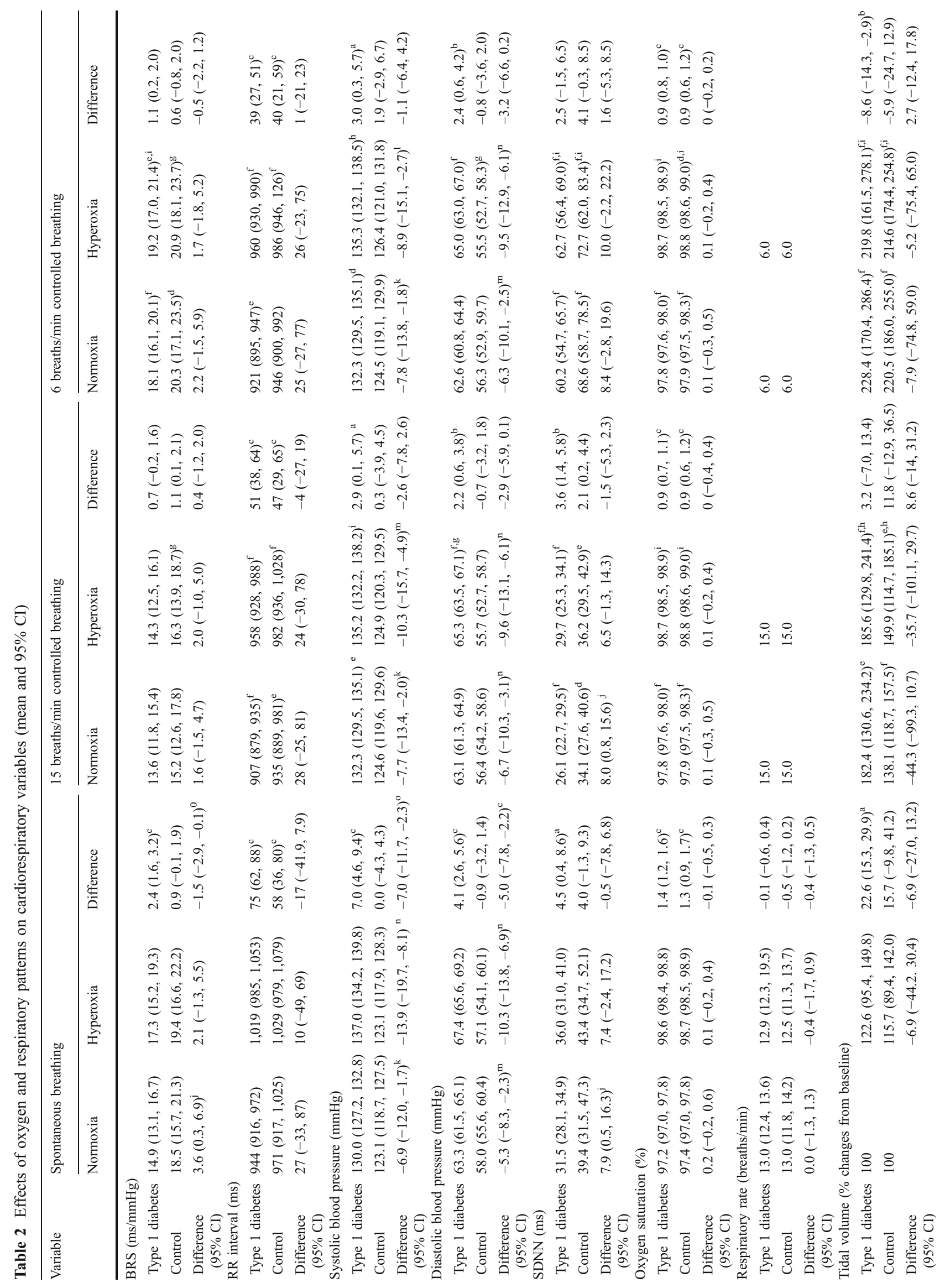




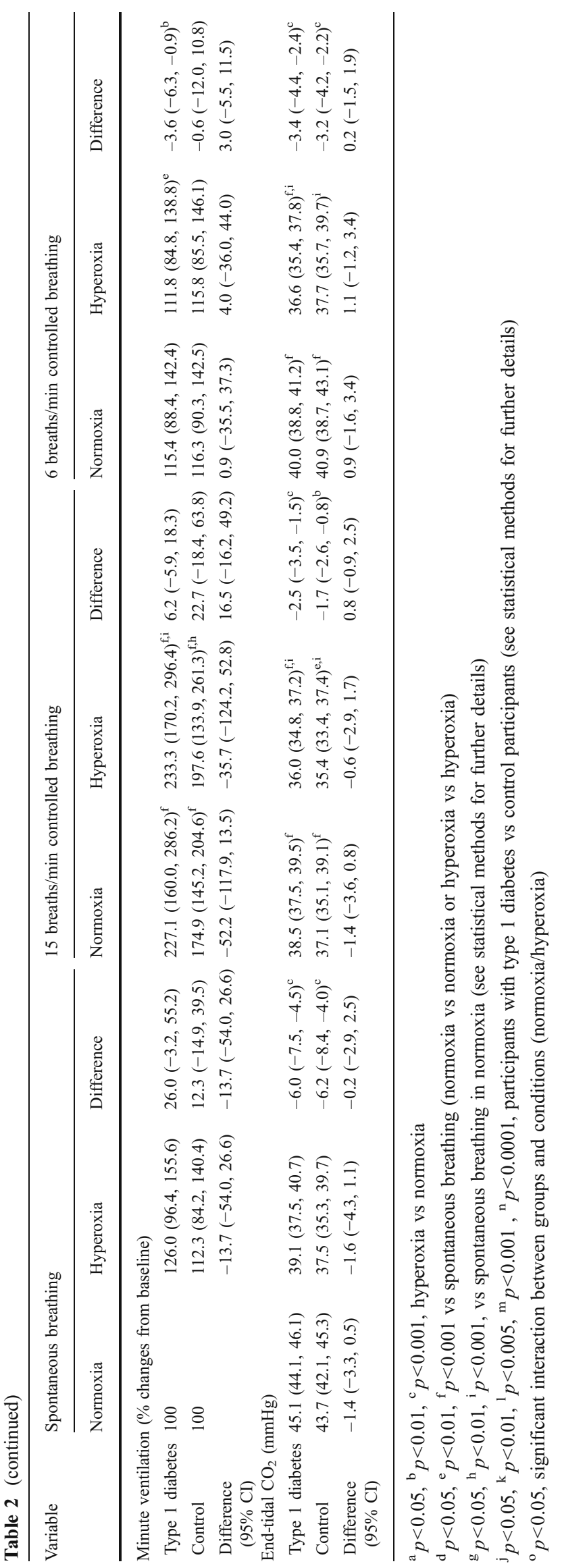


difference in BRS present at rest disappeared (Fig. 1). Controlled breathing at 15 breaths/min induced a significant increase in heart rate (a reduction in RR interval), a reduction in heart rate variability and also a significant reduction in BRS (from $14.8 \pm 0.8$ to $13.5 \pm 0.9 \mathrm{~ms} / \mathrm{mmHg}$ in diabetic participants, and from $18.5 \pm 1.4$ to $15.3 \pm 1.2$ in controls; $p<0.05$ for both). Controlled breathing, and particularly slow breathing, increased oxygen saturation $(p<0.001)$ in normoxia, but while controlled breathing at 15 breaths/min nearly doubled resting ventilation in both groups $(p<0.001)$, slow breathing induced only a modest $(+10 \%)$, non-significant increase in ventilation (Table 2$)$.

Effect of oxygen inhalation Inhalation of $51 / \mathrm{min}$ oxygen increased oxygen saturation $(p<0.001)$ to nearly $99 \%$ on average (Table 2) during spontaneous breathing in both groups. The effect was attenuated but still significant during controlled breathing at 15 breaths/min $(p<0.01$ in diabetic and $p<0.001$ in control participants) and during slow breathing. The attenuation may be explained by the increased level of oxygen saturation due to the controlled or slow breathing per se in normoxia (Table 2).

Oxygen inhalation reduced heart rate (increase in RR interval), and increased heart rate variability (SDNN). Oxygen inhalation increased systolic and diastolic blood pressure and BRS during spontaneous breathing (Table 2), with a similar trend in diabetic and control participants. However, the extent of the change was greater and significant only in the diabetic individuals (Table 2), with a significant $(p<0.05$ or better) interaction effect (groups $\times$ interventions $\times$ breathing) for systolic and diastolic blood pressure. For BRS, there was a significant interaction effect between interventions and groups during spontaneous breathing (Table 2). As a consequence, the significant differences (reported with their confidence limits) observed at baseline between diabetic and control participants increased during oxygen administration $(p<0.001$ for both systolic and diastolic blood pressures), or decreased for BRS ( $p=$ NS; Table 2 and Fig. 1) during spontaneous breathing. The differences with their estimates can be seen in Table 2. It clearly appears that the behaviour of blood pressure and BRS differed between the diabetic and control participants, as shown by a significant interaction. The BRS values obtained during spontaneous breathing approximately predicted the BRS increase induced by oxygen administration $(r=-0.216, p=0.012$; BRS increase from baseline to oxygen $=4.77-0.175 \times$ resting BRS), and showed a reverse effect for higher BRS values; that is, for values $>28 \mathrm{~ms} / \mathrm{mmHg}$ (calculated with the present methodology), oxygen administration would have reduced BRS. This lack of increase with oxygen in fact occurred during slow breathing, when the BRS reached the highest values already in normoxia (Fig. 1). During controlled breathing at 15 breaths/min, the increase in BRS was still present but did not reach statistical significance.

Oxygen administration increased tidal volume during spontaneous breathing in diabetic but not in control participants. However, there was no significant interaction. There was also a trend towards a relative increase in ventilation that was more evident in the diabetic participants. This trend was also confirmed by a reduction in endtidal carbon dioxide. During controlled breathing at 15 breaths/min, oxygen administration induced only minor changes in ventilatory variables (Table 2). Adjusting for age did not modify the results.

The BRS response to oxygen was not influenced by glycaemic control or albuminuria. All these results remained unchanged even after removing the 19 participants treated with drugs affecting angiotensin II.

\section{Discussion}

These results show that the autonomic imbalance and particularly the reduction in BRS of patients with type 1 diabetes can be partially reversed by oxygen, suggesting a possible role of tissue hypoxia.

This observation does not exclude several other factors, such as insulin treatment, hyperglycaemia, antecedent hypoglycaemia, cortisol and poor glycaemic control, from being also relevant. The presence of other possible determinants could explain why the correction remained partial after oxygen administration. However, hypoxia appears to be an important factor that restricts the BRS and modifies the autonomic function of these patients. This finding suggests that the autonomic dysfunction might be part of a more general modification of autonomic reflexes, possibly involving an altered control of ventilation. Previous studies in diabetes showed abnormalities not only in the cardiac vagal regulation, but also in the control of blood pressure [36, 37], microcirculation [38] and respiration $[39,40]$. Respiratory and cardiovascular control are tightly intertwined [41]. Accordingly, any modification of respiratory control will influence cardiovascular control, and vice versa, if the autonomic abnormalities are functional. Conversely, in neural damage, this interaction is minimal or non-existent, as has been shown in patients with severe autonomic neuropathies [42]. Our findings of an important improvement in BRS with oxygen in type 1 diabetes supports our previous findings [7] of a predominantly functional disorder in patients without complications.

Effect of oxygen inhalation Oxygen administration reduces heart rate and increases indices of heart rate variability related to parasympathetic activity [20, 23]. Two studies $[21,22]$ have also reported an increase in BRS in healthy 


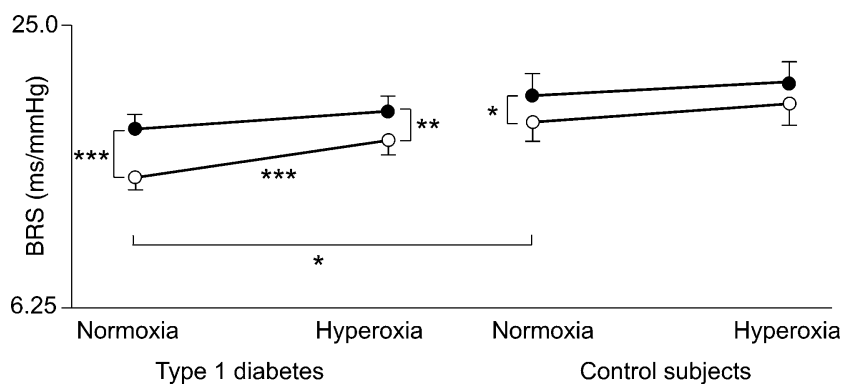

Fig. 1 Effect of oxygen and slow breathing on BRS. Data are expressed as means \pm SEM. White circles, spontaneous breathing; black circles, slow breathing. ${ }^{*} p<0.05, * * p<0.01, * * * p<0.001$. Under spontaneous breathing in ambient conditions (normoxia), BRS is depressed in individuals with type 1 diabetes. However, in hyperoxia, type 1 diabetic participants increase BRS more than controls, and the difference disappears. Slow breathing increases BRS in normoxia in both groups to an extent similar to hyperoxia, and eliminates the difference between diabetic and control participants. Hyperoxia did not further increase the BRS in control participants, indicating that the two effects are probably related

participants and patients with COPD. These findings were interpreted as if oxygen inhalation reduces the chemoreflex activity, even in normoxia. This in turn would increase BRS, as these reflex systems have reciprocal effects. Activation of chemoreflexes normally induces sympathetic activation, an increase in ventilation and a depression of BRS (whereas baroreflex activation is associated with parasympathetic stimulation [41] and opposite effects on ventilation). However, there was no demonstration of any such phenomena in the previous studies on oxygen administration, as ventilation (or chemoreflex) was not evaluated [21, 22]. In our study, we did not measure ventilation or the chemoreflex in absolute values, as the use of a mouthpiece or a face mask would have altered the spontaneous ventilation [33]. However, hyperoxia showed a definite trend towards increased ventilation during spontaneous breathing. This well-documented increase occurs after a transient reduction [24, 25, 43], and has been ascribed to a vagal reflex [43, 44], in connection with an increased generation of oxygen free radicals. There was also some evidence of a more pronounced effect in diabetic participants, probably as a consequence of a greater vagal stimulation induced by oxygen. Unfortunately, the lack of a precise definition of resting ventilation precluded more precise assessment, although the carbon dioxide data confirmed that the increase in ventilation was, at least, as large as in controls. All these findings contrast with the concept of predominant neural damage (that would invariably blunt responses to parasympathetic stimuli) and again suggests an important functional abnormality. Higher preexisting hypoxia and consequent endothelium-related vascular changes are a more logical explanation for the response in patients with type 1 diabetes.
Another interesting and novel finding of the present study is the marked oxygen-induced increase in blood pressure seen in diabetic individuals. Oxygen administration increases blood pressure, probably by a direct local effect. This might have stimulated the BRS and parasympathetic activity. This response is reciprocal to the well-known effect of hypoxia (direct vasodilation, and a reflex increase in sympathetic activity and reduction in BRS). If this were the case, the stronger effect of oxygen seen in type 1 diabetes could again be suggestive of resting tissue hypoxia. An alternative possibility could be an effect of reactive oxygen species on the dysfunctional endothelium typical of diabetes. However, previous findings [45] (and the notion also that hypoxia increases free radicals but causes arterial vasodilation) do not completely support this possibility, which remains open for future research.

Sun et al. [23] were the first and to our knowledge the only group that has tested the effect of hyperoxia in diabetes. They found an increase in heart rate variability in diabetic patients after 4 weeks of hyperbaric hyperoxia. To the extent that their study and our present study can be compared, the results are in complete agreement, but we now provide the first evidence of an improvement in BRS in type 1 diabetes in response to oxygen inhalation. We therefore suggest that the common observation of a low BRS in diabetes may be explained in part by resting tissue hypoxia. The results did not change after removing the participants treated with drugs affecting angiotensin II, confirming no relevant confounding effects of these drugs on BRS [46].

Interaction of oxygen inhalation and respiratory pattern As expected from our previous studies, slow breathing increased BRS in uncomplicated type 1 diabetes [7] and improved oxygen saturation even in normoxia, similar to what has been shown in heart failure [47] or hypoxiadependent diseases [48]. It is possible that the effect of slow breathing in increasing parasympathetic activity and BRS could be mediated by an increase in arterial oxygen pressure, as evidenced by the increased oxygen saturation. Accordingly, the increase in BRS seen during slow breathing was not further augmented by oxygen (Fig. 1). This effect could also have been predicted by the relationship between BRS during spontaneous breathing in normoxia and its changes with oxygen. This suggests that after a maximal value is reached, BRS cannot be further increased (a 'saturation' effect). It is also likely that the high BRS observed during slow breathing prevented a further increase in blood pressure as a result of oxygen in control participants, whereas it could not fully block the increase in diabetic patients, possibly as a consequence of tissue hypoxia and lower resting values. 
The response to oxygen was markedly reduced when breathing was paced at faster respiratory rate $(15 / \mathrm{min})$. This is likely to be due to the very large increase in ventilation induced by paced breathing, and the associated increase in sympathetic activity typically occurring with controlled breathing at faster rates. As such, the increase in ventilation due to voluntary control could have counteracted/blunted the effects oxygen administration.

Implications and conclusions This study has theoretical and practical implications. Diabetic patients are thought to have autonomic abnormalities due to neural damage. In a previous study [7], the low BRS of type 1 diabetic participants was partially restored in the short term by a simple functional manoeuvre (slow breathing), suggesting a functional component. We now provide evidence suggesting that tissue hypoxia may be responsible, in part, for the autonomic dysfunction observed in type 1 diabetes. Hypoxia per se is a potent stimulator of the sympathetic nervous system and of ventilation, which should in turn correct the hypoxia. However, our results indicate that hypoxia persists despite the high sympathetic activity present at rest, and are also suggestive of a disorder in respiratory control.

The importance of uncorrected hypoxia is now being recognised as a key factor in the origin and development of diabetic complications [13, 14], and insufficient compensatory responses to long-term hypoxia have been found in experimental diabetes [49]. Accordingly, subclinical hypoxia could be detected by an improvement in autonomic function after a simple clinical manoeuvre like oxygen administration. Our findings confirm that hyperoxia is not simply a suppression of the chemoreflex stimulus, but provides an additional parasympathetic stimulus, capable of increasing ventilation. The presence of hypoxia, the possible coexistence of abnormal respiratory control and the functional component of these abnormalities highlight the possibility of correction using simple strategies like physical training. Physical training has been shown to improve both respiratory and cardiovascular control in patients with heart failure [50], and is also known to improve cardiovascular and autonomic nervous function in type 2 diabetes $[3,4]$.

In conclusion, resting tissue hypoxia appears as one of several possible causes of functional autonomic abnormalities in patients with type 1 diabetes. These findings may have relevant implications for the understanding of the origin of diabetic complications, as hypoxia may in fact represent a possible link between the autonomic and the other major diabetic complications. Our findings support interventions such as physical activity, and thus may be of practical help in the prevention and management of diabetic complications in patients with type 1 diabetes.
Acknowledgements The study was funded by the Folkhälsan Research Foundation, Wilhelm and Else Stockmann Foundation, Signe and Ane Gyllenberg Foundation, Sigrid Juselius Foundation, Medicinska understödsföreningen Liv och Hälsa, Waldemar von Frenckell Foundation and a special governmental grant for health sciences research (Number 7301). We thank C. Tinelli, San Matteo, Pavia, Italy, for statistical advice.

Duality of interest The authors declare that there is no duality of interest associated with this manuscript.

\section{References}

1. Chalk C, Benstead TJ, Moore F (2007) Aldose reductase inhibitors for the treatment of diabetic polyneuropathy. Cochrane Database Syst Rev, Issue 4, Art. no.: CD004572. doi: 10.1002/ 14651858.CD004572.pub2

2. Schemmel KE, Padiyara RS, D'Souza JJ (2010) Aldose reductase inhibitors in the treatment of diabetic peripheral neuropathy: a review. J Diabetes Complications 24:354-360

3. Loimaala A, Huikuri HV, Koobi T, Rinne M, Nenonen A, Vuori I (2003) Exercise training improves baroreflex sensitivity in type 2 diabetes. Diabetes 52:1837-1842

4. Figueroa A, Baynard T, Fernhall B, Carhart R, Kanaley JA (2007) Endurance training improves post-exercise cardiac autonomic modulation in obese women with and without type 2 diabetes. Eur J Appl Physiol 100:437-444

5. Raupach T, Bahr F, Herrmann P et al (2008) Slow breathing reduces sympathoexcitation in chronic obstructive pulmonary disease. Eur Respir J 32:387-392

6. Naughton MT, Floras JS, Rahman MA, Jamal M, Bradley TD (1998) Respiratory correlates of muscle sympathetic nerve activity in heart failure. Clin Sci (Lond) 95:277-285

7. Rosengård-Bärlund M, Bernardi L, Fagerudd J et al (2009) Early autonomic dysfunction in type 1 diabetes: a reversible disorder? Diabetologia 52:1164-1172

8. Adler GK, Bonyhay I, Failing H, Waring E, Dotson S, Freeman R (2009) Antecedent hypoglycemia impairs autonomic cardiovascular function: implications for rigorous glycemic control. Diabetes 58:360-366

9. Broadley AJ, Korszun A, Abdelaal E et al (2005) Inhibition of cortisol production with metyrapone prevents mental stressinduced endothelial dysfunction and baroreflex impairment. J Am Coll Cardiol 146:344-350

10. Larsen JR, Sjøholm H, Berg TJ et al (2004) Eighteen years of fair glycemic control preserves cardiac autonomic function in type 1 diabetes. Diabetes Care 27:963-966

11. Hoffman RP, Hausberg M, Sinkey CA, Anderson EA (1999) Hyperglycemia without hyperinsulinemia produces both sympathetic neural activation and vasodilation in normal humans. J Diabetes Complications 13:17-22

12. Scherrer U, Sartori C (1997) Insulin as a vascular and sympathoexcitatory hormone: implications for blood pressure regulation, insulin sensitivity, and cardiovascular morbidity. Circulation 96:4104-4113

13. Miyata T, van Ypersele de Strihou C (2010) Diabetic nephropathy: a disorder of oxygen metabolism? Nature Rev Nephrol 6:83-95

14. Heyman SN, Khamaisi M, Rosen S, Rosenberger C (2008) Renal parenchymal hypoxia, hypoxia response and the progression of chronic kidney disease. Am J Nephrol 28:998-1006

15. Williamson JR, Chang K, Frangos M et al (1993) Hyperglycemic pseudohypoxia and diabetic complications. Diabetes 42:801-813

16. Bernardi L, Passino C, Spadacini G et al (1998) Cardiovascular autonomic modulation and activity of carotid baroreceptors at altitude. Clin Sci (Lond) 95:565-573 
17. La Rovere MT, Bigger JT Jr, Marcus FI, Mortara A, Schwartz PJ (1998) Baroreflex sensitivity and heart-rate variability in prediction of total cardiac mortality after myocardial infarction. ATRAMI (Autonomic Tone and Reflexes After Myocardial Infarction) Investigators. Lancet 351:478-484

18. Johansson M, Gao SA, Friberg P et al (2007) Baroreflex effectiveness index and baroreflex sensitivity predict all-cause mortality and sudden death in hypertensive patients with chronic renal failure. J Hypertens 25:163-168

19. Sykora M, Diedler J, Turcani P, Hacke W, Steiner T (2009) Baroreflex: a new therapeutic target in human stroke? Stroke 40: e678-e682

20. Lund VE, Kentala E, Scheinin H et al (1999) Heart rate variability in healthy volunteers during normobaric and hyperbaric hyperoxia. Acta Physiol Scand 167:29-35

21. Waring WS, Thomson AJ, Adwani SH et al (2003) Cardiovascular effects of acute oxygen administration in healthy adults. $\mathrm{J}$ Cardiovasc Pharmacol 42:245-250

22. Bartels MN, Gonzalez JM, Kim W, de Meersman RE (2000) Oxygen supplementation and cardiac-autonomic modulation in COPD. Chest 118:691-696

23. Sun TB, Yang CC, Kuo TB (2006) Effect of hyperbaric oxygen on cardiac neural regulation in diabetic individuals with foot complications. Diabet Med 23:360-366

24. Marczak M, Pokorski M (2004) Oxygen breathing and ventilation. J Physiol Pharmacol 55:127-134

25. Budzinska K, Ilasz R (2008) Superoxide dismutase mimetic modulates hyperoxic augmentation of the diaphragmatic response to poikilocapnic hypoxia in non-vagotomized rats. J Physiol Pharmacol 59(Suppl 6):163-172

26. Tesfaye S, Boulton AJ, Dyck PJ et al (2010) Diabetic neuropathies: update on definitions, diagnostic criteria, estimation of severity, and treatments. Diabetes Care 33:2285-2293

27. Laude D, Elghozi JL, Girard A et al (2004) Comparison of various techniques used to estimate spontaneous baroreflex sensitivity (the EuroBaVar study). Am J Physiol (Regul Integr Comp Physiol) 286:R226-R231

28. Bernardi L, De Barbieri G, Rosengård-Bärlund M, Mäkinen VP, Porta C, Groop PH (2010) New method to measure and improve consistency of baroreflex sensitivity values. Clin Auton Res 20:353-361

29. Bertinieri G, Di Rienzo M, Cavallazzi A, Ferrari AU, Pedotti A, Mancia G (1985) A new approach to analysis of the arterial baroreflex. J Hypertens 3:S79-S81

30. Pagani M, Somers V, Furlan R et al (1988) Changes in autonomic regulation induced by physical training in mild hypertension. Hypertension 12:600-610

31. Pinna GD, Maestri R (2001) Reliability of transfer function estimates in cardiovascular variability analysis. Med Biol Eng Comput 39:338-347

32. Tobin MJ, Jenouri G, Lind B, Watson H, Schneider A, Sackner MA (1983) Validation of respiratory inductive plethysmography in patients with pulmonary disease. Chest 83:615-620

33. Askanazi J, Silverberg PA, Foster RJ, Hyman AI, Milic-Emili J, Kinney JM (1980) Effects of respiratory apparatus on breathing pattern. J Appl Physiol 48:577-580
34. American Diabetes Association (2006) Standards of medical care in diabetes-2006. Diabetes Care 29:S4-S42

35. Bruning JL, Kintz BL (1968) Computational handbook of statistics. Scott Foresman, Glenview

36. Lefrandt JD, Hoogenberg K, van Roon AM, Dullaart RP, Gans RO, Smit AJ (1999) Baroreflex sensitivity is depressed in microalbuminuric type I diabetic patients at rest and during sympathetic manoeuvres. Diabetologia 42:1345-1349

37. Frattola A, Parati G, Gamba P et al (1997) Time and frequency domain estimates of spontaneous baroreflex sensitivity provide early detection of autonomic dysfunction in diabetes mellitus. Diabetologia 40:1470-1475

38. Bernardi L, Rossi M, Leuzzi S et al (1997) Reduction of $0.1 \mathrm{~Hz}$ microcirculatory fluctuations as evidence of sympathetic dysfunction in insulin-dependent diabetes. Cardiovasc Res 34:185-191

39. Tantucci C, Scionti L, Bottini P et al (1997) Influence of autonomic neuropathy of different severities on the hypercapnic drive to breathing in diabetic patients. Chest 112:145-153

40. Mancini M, Filippelli M, Seghieri G et al (1999) Respiratory muscle function and hypoxic ventilatory control in patients with type I diabetes. Chest 115:1553-1562

41. Francis DA, Coats JS, Ponikowski P (2000) Chemoreflexbaroreflex interactions in cardiovascular disease. In: Bradley TD, Floras JS (eds) Sleep apnea. Implication in cardiovascular and cerebrovascular disease. Marcel Dekker, New York, pp 261-283

42. Bernardi L, Hilz M, Stemper B, Passino C, Welsch G, Axelrod FB (2003) Respiratory and cerebrovascular responses to hypoxia and hypercapnia in familial dysautonomia. Am J Respir Crit Care Med 167:141-149

43. Ruan T, Ho CY, Kou YR (2003) Afferent vagal pathways mediating respiratory reflexes evoked by ROS in the lungs of anesthetized rats. J Appl Physiol 94:1987-1998

44. Dean JB, Mulkey DK, Henderson RA, Potter SJ, Putnam RW (2004) Hyperoxia, reactive oxygen species, and hyper-ventilation: oxygen sensitivity of brain stem neurons. J Appl Physiol 96:784791

45. Thomson AJ, Drummond GB, Waring SW, Webb DJ, Maxwell SRJ (2006) Effects of short-term isocapnic hyperoxia and hypoxia on cardiovascular function. J Appl Physiol 101:809-816

46. de Tommasi E, Iacoviello M, Romito R et al (2003) Comparison of the effect of valsartan and lisinopril on autonomic nervous system activity in chronic heart failure. Am Heart J 146:E17

47. Bernardi L, Spadacini G, Bellwon J, Hajric R, Roskamm H, Frey AW (1998) Effect of breathing rate on oxygen saturation and exercise performance in chronic heart failure. Lancet 351:13081311

48. Keyl C, Schneider A, Gamboa A et al (2003) Autonomic cardiovascular function in high-altitude Andean natives with chronic mountain sickness. J Appl Physiol 94:213-219

49. Catrina SB, Okamoto K, Pereira T, Brismar K, Poellinger L (2004) Hyperglycemia regulates hypoxia-inducible factor-1alpha protein stability and function. Diabetes 53:3226-3232

50. Coats AJS, Adamopoulos S, Radaelli A et al (1992) Controlled trial of physical training in chronic heart failure. Exercise performance, hemodynamics, ventilation, and autonomic function. Circulation 85:2119-2131 